\title{
SOLUTION AND COST ANALYSIS OF GENERAL MULTI-CHANNEL AND MULTI-STAGE FILTERING CIRCUITS
}

\author{
M. Alper Kutay ${ }^{1}$, Hakan Özaktaş ${ }^{1}$, M. Fatih Erden ${ }^{2}$, Haldun M. Ozaktas ${ }^{1}$, Orhan Arıkan ${ }^{1}$ \\ ${ }^{1}$ Bilkent University, Faculty of Engineering, TR-06533 Bilkent, Ankara, Turkey \\ ${ }^{2}$ Tampere University of Technology, P.O. Box 553, SF-33101, Tampere, Finland \\ kutay@ee.bilkent.edu.tr
}

\begin{abstract}
The fractional Fourier domain multi-channel and multistage filtering configurations that have been recently proposed enable us to obtain either exact realizations or useful approximations of linear systems or matrixvector products in many different applications. We discuss the solution and cost analysis for these configurations. It is shown that the problem can be reduced to a least squares problem which can be solved with fast iterative techniques.
\end{abstract}

\section{INTRODUCTION}

In many applications of digital and optical signal processing, it is desired to implement linear systems of the form $g(u)=\int H\left(u, u^{\prime}\right) f\left(u^{\prime}\right) d u^{\prime}$. Such systems take the form of a matrix-vector product when discretized: $g_{k}=\sum_{n=1}^{N} H_{k n} f_{n}$ or $\mathbf{g}=\mathbf{H f}$. This may either represent a system which is inherently discrete or may constitute an approximation of a continuous system.

Linear shift-invariant systems are characterized by kernels of the special form $H\left(u, u^{\prime}\right)=h\left(u-u^{\prime}\right)$ or $H_{k n}=h_{k-n}$. These systems correspond to convolution in the time or space domain and multiplication with a filter function in the Fourier domain. Although the use of shift-invariant (convolution-type) systems are convenient in many applications, sometimes their use is inappropriate or at best a crude approximation.

In a variety of applications, greater flexibility and performance can be achieved at no additional cost, by filtering in fractional rather than ordinary Fourier domains (Fig. 1a) $[2,3,4,5,6]$. The ath order fractional Fourier transform $\mathcal{F}^{a}$ is the generalization of the ordinary Fourier transform, such that $a=1$ corresponds to the ordinary Fourier transform and $a=0$ corresponds to the identity operation $[1,2,7]$. Thus, when $a=1$, the filtering scheme in Fig. 1a corresponds to ordinary Fourier domain filtering (shift-invariant or convolution-type systems). When $a=0$, it corresponds to direct multiplication by $h(u)$ in the time domain. The costs of both digital and optical implementations of fractional Fourier domain filtering are the same as that of ordinary Fourier domain filtering $[3,8]$.

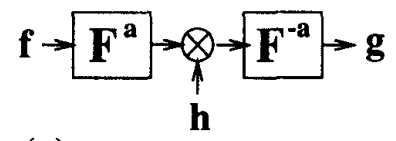

(a)
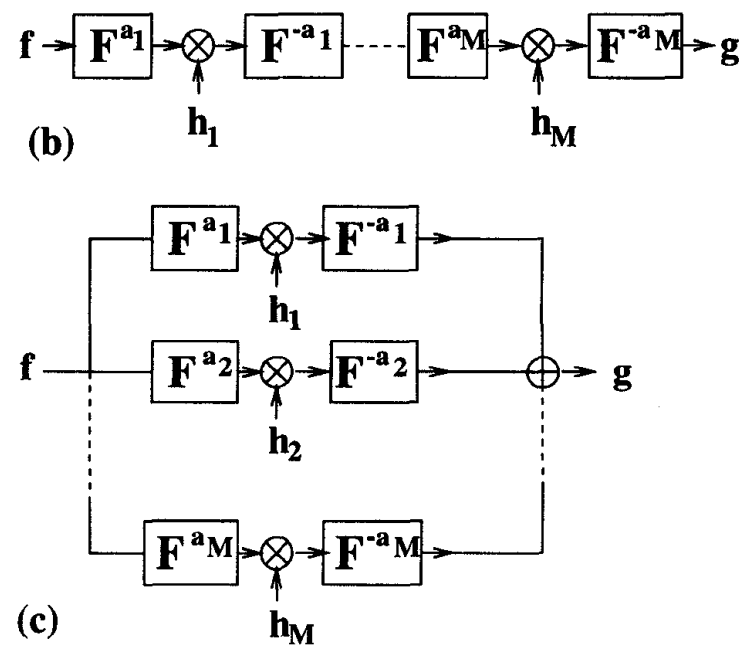

Figure 1: (a) ath order fractional Fourier domain filtering. (b) Multi-stage (serial) filtering. (c) Multichannel (parallel) filtering.

Further generalizations of the concept of fractional Fourier domain filtering have been suggested. These have been referred to as multi-stage (or repeated or serial) filtering in fractional Fourier domains, and multichannel (or parallel) filtering in fractional Fourier domains, In the multi-stage system (Fig.1b) [2, 5, 9], the input is first transformed into the $a_{1}$ th domain where it 
is multiplied by a filter $h_{1}(u)$. The result is then transformed back into the original domain. This process is repeated $M$ times. In the more recently suggested multi-channel filter structure (Fig.1c) $[10,11]$, the inputs of all channels are identical and their outputs are added together. For each channel $k$, the input is first transformed to the $a_{k}$ th domain where it is multiplied with a filter $h_{k}(u)$. The result is then transformed back to the original (time) domain.

In previous works $([2,5,9,10,11])$, the matrices involved in each configuration were assumed to be square matrices of full rank. In this work a generalization of the formulation is carried out for arbitrary rectangular matrices of arbitrary dimensions and rank.

\section{MULTI-STAGE AND MULTI-CHANNEL FILTERING}

In discrete-time notation, the outputs $g_{s}$ and $g_{p}$ of the serial and parallel configurations of Fig.1b and Fig.1c are related to the input $f$ by the relations:

$$
\begin{array}{r}
g_{s}=\left[\mathbf{F}^{-a_{M}} \boldsymbol{\Lambda}_{M} \ldots \mathbf{F}^{a_{2}-a_{1}} \boldsymbol{\Lambda}_{1} \mathbf{F}^{a_{1}}\right] f, \\
g_{p}=\left[\sum_{k=1}^{M} \mathbf{F}^{-a_{k}} \boldsymbol{\Lambda}_{k} \mathbf{F}^{a_{k}}\right] f,
\end{array}
$$

where $\mathbf{F}^{a_{j}}$ represents the $a_{j}$ th order fractional Fourier transform matrix $[12,13]$, and $\boldsymbol{\Lambda}_{j}$ denote the diagonal matrix corresponding to multiplication by the filter function $h_{k}[j]$. The above may also be expressed as $g=\mathbf{T} f$ where $\mathbf{T}$ is the matrix representing the overall filtering configuration. In previous work $([2,5,9,10])$, $\mathbf{T}$ was assumed to be a square matrix of full rank. In this work we provide a generalization of both the formulation and cost analysis to arbitrary rectangular matrices $\mathrm{T}$ of dimension $N_{g} \times N_{f}$ of arbitrary rank $R$. In the multi-channel configuration, the dimensions of $\mathbf{F}^{-a_{k}}, \boldsymbol{\Lambda}_{k}$, and $\mathbf{F}^{a_{k}}$ become $N_{g} \times N_{g}, N_{g} \times N_{f}$, $N_{f} \times N_{f}$. To avoid confusion, in the rest of the paper we will use $\mathbf{F}_{N}^{a_{k}}$ to denote the $a_{k}$ th order fractional Fourier transform matrix with dimensions $N \times N$. In the multi-stage case there exists a greater flexibility in choosing the dimensions of the intermediate filter matrices $\boldsymbol{\Lambda}_{k}$. A natural choice is to taper the dimensions of $\boldsymbol{\Lambda}_{k}$ gradually from $N_{f}$ to $N_{g}$ as $k$ goes from 1 to $M$.

In a typical application we are given a linear system matrix $\mathbf{H}$ which we desire to implement (which may, for instance, be the optimal recovery operator of a signal restoration problem). Then, we seek the transform orders $a_{k}$ and filters $h_{k}[j]$ such that the resulting matrix $\mathbf{T}$ (as given by Eqns. 1 and 2) is as close as possible to $\mathbf{H}$ according to some specified criteria, such as Froebenius norm: $\|\mathbf{T}-\mathbf{H}\|_{\mathrm{F}}$. Alternatively, it is possible to take Eqn 1 or 2 as a constraint on the form of the linear matrix $\mathrm{H}$ to be employed in a specific application such as restoration, recovery, denoising, etc. Given a specific optimization criteria, such as minimum meansquare estimation error, we seek the optimal values of $a_{k}$ and $h_{k}[j]$ such that the given criteria is optimized.

In the multi-channel case, regardless of which of these approaches we take, the problem of determining the optimal filter coefficients can be exactly solved since the overall kernel $\mathbf{T}$ depends linearly on the filter coefficients $h_{k}[j]$ as follows:

$$
\mathbf{T}=\sum_{k=1}^{M} \sum_{j=1}^{N} h_{k}[j] \hat{\mathbf{T}}_{k j}
$$

where $N \equiv \min \left(N_{f}, N_{g}\right)$. The dimensions of the matrices $\hat{\mathbf{T}}_{k j}$, indexed by $k j$, are the same as the dimensions of $\mathbf{T}\left(N_{g} \times N_{f}\right)$. These matrices play the role of a family of "basis matrices" which are used to construct the matrix $\mathcal{T}$. It can be shown that their elements $\hat{\mathcal{T}}_{k j}[m, n]$ are given by

$$
\hat{\mathcal{T}}_{k j}[m, n]=\mathcal{F}_{N_{g}}^{-a_{k}}[m, j] \mathcal{F}_{N_{f}}^{a_{k}}[j, n],
$$

where $\mathcal{F}_{N_{g}}^{-a_{k}}$ and $\mathcal{F}_{N_{f}}^{a_{k}}$ are fractional Fourier transform matrices of dimension $N_{g}$ and $N_{f}$ respectively.

The objective is to choose the $N M$ filter coefficients $h_{k}[j]$ ( $N$ coefficients in each of $M$ filters) so that the resulting linear system $\mathbf{T}$ is optimal according to some criteria. For instance, if we wish to minimize $\|\mathbf{T}-\mathbf{H}\|_{F}$, where $\mathbf{H}$ is a specified matrix, the problem can be exactly posed as a least-squares optimization problem leading to an associated set of normal equations or which can be solved with other standard techniques. To see this, it is necessary to first "vectorize" the above equations. Let $\underline{\mathbf{T}}$ denote the $N_{g} N_{f} \times 1$ vector obtained by stacking the columns of $\mathbf{T}$ on top of each other, and let $\hat{\mathbf{T}}_{k j}$ denote the $N_{g} N_{f} \times 1$ vector obtained by stacking the columns of $\hat{\mathbf{T}}_{k j}$ on top of each other. Finally, let $\underline{\mathbf{h}}$ denote the $M N \times 1$ matrix obtained by stacking the $M$ filters $h_{1}[j], h_{2}[j], \ldots, h_{N}[j]$ on top of each other. With these conventions, we obtain

$$
\underline{\mathbf{T}}[p]=\sum_{q=1}^{M N} \underline{\hat{\mathbf{T}}}_{q}[p] \underline{\mathbf{h}}[q] \quad p=1,2, \ldots, N_{g} N_{f}
$$

where the indice $q$ also follows a column ordering over the two indices $k j$. 'This equation can also be written in matrix form as

$$
\underline{\mathbf{T}}=\left[\underline{\mathbf{T}}_{1} \underline{\mathbf{T}}_{2} \ldots \underline{\mathbf{T}}_{M N}\right] \underline{\mathbf{h}} \equiv \underline{\hat{\mathbf{T}}} \underline{\mathbf{h}}
$$

where the new $N_{g} N_{f} \times M N$ matrix $\underline{\underline{\mathbf{T}}}$ has been defined. 
Now, we are finally able to state our problem in standard form as follows: Minimize the mean-square difference $\|\underline{\mathbf{T}}-\underline{\mathbf{H}}\|^{2}$ between the desired $\underline{\mathbf{H}}$ and $\underline{\mathbf{T}}=$ $\hat{\hat{\mathbf{T}} \mathbf{h}}$. This is a standard least squares problem and can be solved in a number of ways. The filter vector $\underline{\mathbf{h}}$ which minimizes $\|\underline{\mathbf{H}}-\underline{\hat{\hat{\mathbf{T}}} \mathbf{h}}\|^{2}$ is known to satisfy the so-called normal equations associated with the least squares problem:

$$
\underline{\hat{\mathbf{T}}}^{\mathbf{H}} \underline{\mathbf{H}}=\underline{\hat{\mathbf{T}}}^{\mathbf{H}} \underline{\hat{\mathbf{T}}} \underline{\mathbf{h}}
$$

where $\underline{\hat{\mathbf{T}}}^{\mathbf{H}}$ is the Hermitian transpose of $\hat{\hat{\mathbf{T}}}$.

In the multi-stage case, the overall kernel $\mathbf{T}$ depends nonlinearly on the filter coefficients $h_{k}[j]$, so that solution of the optimization problem arising in this case is much more difficult. (Nevertheless an iterative approach has been successfully applied to this problem $[5,9]$.)

The $M$-channel or $M$-stage filtering configuration has about $M N$ degrees of freedom, as opposed to general linear systems which have $N_{g} N_{f}$ degrees of freedom and shift-invariant systems which have about $N$ degrees of freedom. These configurations interpolate between general linear systems and shift-invariant systems both in terms of cost and flexibility. If we choose $M$ to be small, cost and flexibility are both low; $M=1$ corresponds to single-stage filtering. If we choose $M$ larger, cost and flexibility are both higher; as $M$ approaches $N$, the number of degrees of freedom approaches that of a general linear system. We show that exactly $M=N$ filters are necessary and sufficient to implement an arbitrary general linear system matrix exactly (with zero error) in the multi-channel case. Likewise, $M=N+1$ filters are necessary and sufficient in the multi-stage case. In practice, most matrices are not wholly arbitrary and exhibit some kind of internal structure, although that structure may not be easy to identify or characterize. As will be illustrated by the examples, there are many applications in which acceptable or useful approximations to given linear systems are possible with small or moderate values of $M \ll N$, which as discussed next result in considerable cost savings.

A general cost (complexity) analysis has been undertaken for both digital and optical implementations in the form of multi-channel and multi-stage filtering configurations, in comparison to direct implementations in the form of general linear systems. While we have considered the general case where the linear system matrix $\mathbf{H}$ we wish to approximately implement has dimensions $N_{g} \times N_{f}$ and rank $R$, here we only present the simplest case of digital implementation of full rank square matrices (Fig. 2). The number of channels or

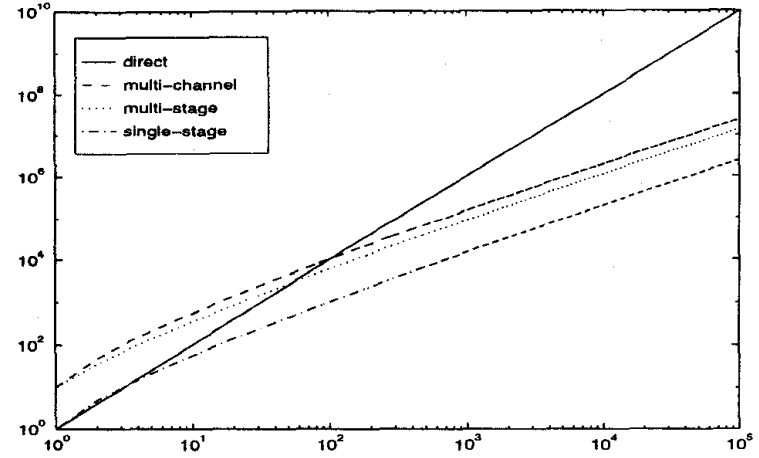

Figure 2: Cost comparison of exact and approximate implementations of linear systems.

stages $M=10$ assumed in these plots has been shown to be sufficient to obtain useful approximations to the desired linear system matrix in a variety of applications.

\section{EXAMPLES}

As a simple example, we first consider restoration of images blurred by a space-varying local point spread function using (Fig.3). The mean-square estimation error is $12 \%$ in the multi-stage case and $16 \%$ in the multi-channel case with $M=4$, and $4 \%$ in the multistage case and $6 \%$ in the multi-channel case with $M=$ 8. Ordinary Fourier domain filtering gives very poor results, resulting in an error of $34 \%$.

Next we consider the problem of denoising a signal consisting of $Q$ chirp-like components in additive white Gaussian noise. We assume that the rates of the chirps are known within an accuracy of $5 \%$ but that their time shifts are unknown. For $Q=6$ and an initial $S N R=0.1$, the multi-channel configuration results in a mean-square estimation error of $2.6 \%$ with $M=6$. This corresponds to an SNR improvement of $25.8 \mathrm{~dB}$. These results represent significant improvements with respect to single domain filtering but are much cheaper to implement than general linear filtering.

\section{REFERENCES}

[1] D. Mendlovic and H. M. Ozaktas, "Fractional Fourier transforms and their optical implementation: I," J. Opt. Soc. Am. A, 10, pp. 1875 1881,1993 .

[2] H. M. Ozaktas, B. Barshan, D. Mendlovic, and L. Onural, Convolution, filtering, and multiplexing in fractional Fourier domains and their relation to 


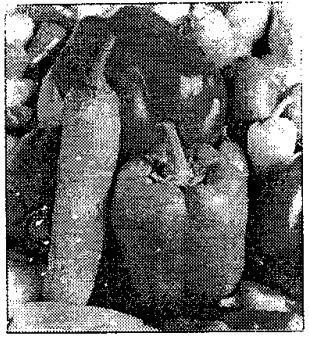

a

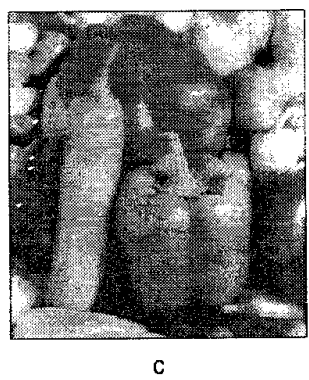

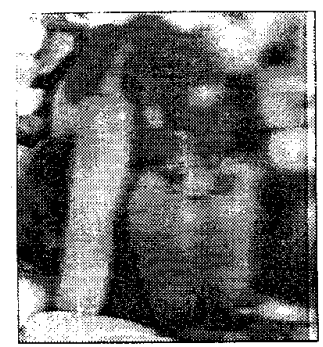

b

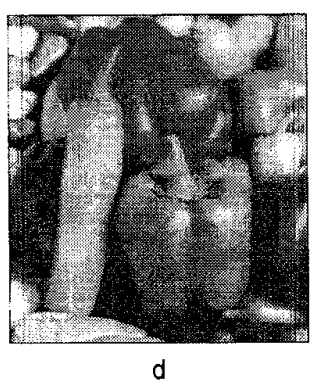

Figure 3: (a) Original $256 \times 256$ image. (b) Blurred image. (c). Restored by multi-stage filtering $(M=8)$. (d) Restored by multi-channel filtering $(M=8)$.

chirp and wavelet transforms. J. Opt. Soc. Am. A 11, pp. 547-559, 1994.

[3] M. A. Kutay, H. M. Ozaktas, O. Arıkan, and L. Onural, Optimal filtering in fractional Fourier domains. IEEE Trans. Sig. Proc. 45, pp. 1129-1143, 1997.

[4] M. A. Kutay and H. M. Ozaktas, "Optimal image restoration with the fractional Fourier transform," J. Opt. Soc. Am. A 15, pp. $825-834,1998$.

[5] M. F. Erden and H. M. Ozaktas. Synthesis of general linear systems with repeated filtering in consecutive fractional Fourier domains. To appear in J. Opt. Soc. Am. A.

[6] Z. Zalevsky and D. Mendlovic, Fractional Wiener filter. Applied Optics, 35, pp. 3930-3936, 1996.

[7] L. B. Almeida, The fractional Fourier transform and time-frequency representations. IEEE Trans. Sig. Proc. 42, pp. 3084 - 3092, 1994.

[8] H. M. Ozaktas, O. Arkan, M. A. Kutay, and G. Bozdagi, Digital computation of the fractional Fourier transform. IEEE Trans. Sig. Proc. 44, pp. 2141 - 2150, 1996.

[9] M. F. Erden. Repeated Filtering in Consecutive Fractional Fourier Domains. Ph.D. Thesis, Bilkent University, Ankara, 1997.

[10] M. A. Kutay, M. F. Erden, H. M. Ozaktas, O. Arıkan, Ç. Candan, and Ö. Güleryüz, "CostEfficient approximation of linear systems with repeated and multi-channel filtering configurations", Proceedings of IEEE ICASSP 1998, vol. 6, pp. 3433-3437, May 12-15, Seattle, 1998.

[11] M. A. Kutay, M. Fatih Erden, H. M. Ozaktas, O. Arıkan, Ö. Güleryüz and C. Candan, SpaceBandwidth Efficient Realizations of Linear Systems Optics Letters, 23, pp. 1069-1071, 1998.

[12] S. C. Pei, C. C. Tseng, M. H. Yeh, and J. J. Shyu, "Discrete fractional Hartley and Fourier transforms," IEEE Trans. on Circuits and Systems-II, 1998.

[13] C. Candan. The discrete fractional Fourier Transformation. MSc. Theises, Bilkent University, Ankara, July 1998. 